\title{
Strangeness in the nucleon and the ratio of proton-to-neutron neutrino-induced quasi-elastic yield
}

\author{
E. Kolbe \\ Institut für Physik der Universität Basel \\ Klingelbergstrasse 82 \\ CH-4056 Basel, Switzerland \\ S. Krewald \\ Institut für Kernphysik \\ Forschungsanlage Jülich \\ D-52425 Jülich, Germany \\ and \\ H. Weigel \\ Institut für Theoretische Physik \\ Universität Tübingen \\ D-72076 Tübingen, Germany
}

December 1996

\begin{abstract}
The electroweak form factors of the nucleon as obtained within a three flavor pseudoscalar vector meson soliton model are employed to predict the ratio of the proton and neutron yields from ${ }^{12} \mathrm{C}$, which are induced by quasi-elastic neutrino reactions. These predictions are found to vary only moderately in the parameter space allowed by the model. The antineutrino flux of the up-coming experiment determining this ratio was previously overestimated. The corresponding correction is shown to have only a small effect on the predicted ratio. However, it is found that the experimental result for the ratio crucially depends on an accurate measurement of the energy of the knocked out nucleon.
\end{abstract}




\section{Introduction}

By means of a continuum random phase approximation (RPA) [1, 2] it has been shown [3, 4] that the ratio $R_{y}$ of the proton-to-neutron neutrino-induced quasi-elastic yields from an isoscalar nucleus provides a relation between the nucleon matrix elements of the strange quark vector and axial vector currents $\left\langle N\left|\bar{s} \gamma_{\mu} s\right| N\right\rangle$ and $\left\langle N\left|\bar{s} \gamma_{\mu} \gamma_{5} s\right| N\right\rangle$, respectively. This

is especially interesting because it allows one to determine the singlet matrix element $g_{A}^{(0)}$ of the axial vector current (the so-called proton spin puzzle [5]) without assuming flavor $S U(3)$ symmetry. This symmetry is commonly employed to relate matrix elements of currents which conserve the flavor charges to those which change this quantum number. The latter are measured in semi-leptonic hyperon decays.

The purpose of the present paper is three-fold. Firstly, we will update the earlier calculations [3, 4] by substituting more accurate energy spectra for the LAMPF neutrino and antineutrino beam which recently became available [6]. This spectrum enters the computation of the quasi elastic yields by folding it into the cross-section for the (anti) neutrino - nucleus reaction [2]. In the second part we will adopt the $S U(3)$ Skyrme model with vector mesons [7] to compute the relevant momentum dependent form factors of the nucleon. This makes possible a prediction for $R_{y}$ because these form factors serve as the

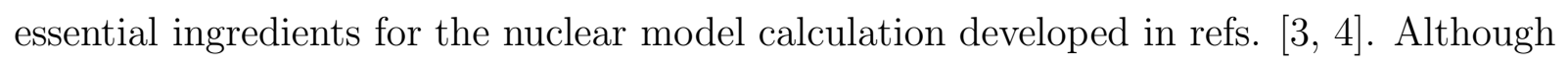
the incorporation of vector meson fields into the $S U(3)$ Skyrme model represents a severe complication [8] these fields are unavoidable for a realistic description of static baryon properties. In particular these fields account for short range effects, which are particularly important to gain a realistic, non-zero value for $g_{A}^{(0)}[9]$. The simple Skyrme model of pseudoscalars predicts $g_{A}\left(q^{2}\right) \equiv 0$ [10]. Such short range components are also necessary to gain a non-zero result for the neutron-proton mass difference [11]. On the basis that this model reproduces the experimental two-flavor form factors only within an error of 20$30 \%$ (which already represents an improvement compared with the pure $S U(3)$ Skyrme model) one might want to argue that other approaches [12] may be more appropriate to compute strange form factors. However, this model is unique in the sense that it treats vector and axial vector currents simultaneously and contains a consistent and systematic treatment of symmetry breaking effects. The resulting predictions for $R_{y}$ turn out to be quite insensitive to the model parameters. This indicates that the strange form factors may independently be considered from those in the two flavor subspace. A recent study [13] has shown that the ratio $R_{y}$ is not very sensitive to the nuclear model but significantly depends on the prediction for $\left\langle N\left|\bar{s} \gamma_{\mu} \gamma_{5} s\right| N\right\rangle$. In this respect a precise measurement of $R_{y}$ by the LSND-Collaboration at LAMPF [14] will be discriminating on the nucleon model. In section 3 we will briefly review the computation of the relevant form factors in this 
model after having outlined (in section 2) their relevance for the (anti)neutrino scattering off nuclei in the context of the continuum RPA.

Finally we will point out a possible source of uncertainty in the experiment associated with the sensitive dependence of $R_{y}$ on the energy thresholds for the integrated proton and neutron yields. The corresponding results together with a comprehensive discussion will be presented in section 4 .

\section{The $\nu-\bar{\nu}$ nuclei reaction}

At low energies (compared to the electro-weak scale) the interaction of neutrinos with matter is described by the current-current interaction introducing the Fermi coupling constant $G_{F}$

$$
\mathcal{L}_{\text {eff }}=-\frac{G_{F}}{\sqrt{2}}\left(j_{\mu}^{(+)} J^{\mu(-)}+j_{\mu}^{(0)} J^{\mu(0)}+\text { h. c. }\right) .
$$

Here $j_{\mu}^{(a)}$ denotes the leptonic part of the electro-weak current円 while $J^{\mu(a)}$ represents the hadronic counterpart. Of course, these currents break parity in a maximal way, i.e. $j_{\mu}^{(a)}=v_{\mu}^{(a)}-a_{\mu}^{(a)}$ with $v$ and $a$ referring to the vector and axial-vector currents. The hadronic (axial)vector currents $(V$ and $A$ ) are defined similarly.

To describe neutrino scattering off nuclei in a perturbative treatment of $\mathcal{L}_{\text {eff }}$ we will employ the continuum RPA approach (as a mean field model) of ref. [1, 2] in order to compute matrix elements of $J^{\mu(a)}$ when sandwiched between nuclei states?. In this nuclear model the interaction between the constituents of the nucleus is described by combining the usual RPA treatment with a correct description of the particle states in the continuum, i.e. the excited many-body states are coherent superpositions of one-particle-one-hole $(1 p$ $1 h$ ) excitations obeying the proper Coulomb boundary conditions for scattering states. For details on this approach we refer to the literature [1, 2]. Here we restrict ourselves to a brief description of its basic features and ingredients. The continuum RPA provides a good description of the nuclear ground state while the excited states are generic continuum states possessing a $1 p$-1h structure. Final state interactions are accounted for by a realistic (finite range) residual interaction derived from the Bonn meson exchange potential 15, 16]. It should also be mentioned that this treatment assumes that the reactions proceed predominantly via the knocked out nucleon. As the main result of the continuum RPA approach the scattering cross section of the (anti)neutrino nuclei reaction is expressed in terms of the nucleon matrix elements

$$
\left\langle N\left|J^{\mu(a)}\right| N\right\rangle, \quad a=0, \ldots, 8,
$$

\footnotetext{
${ }^{1}$ This shorthand notation is assumed to include the dependence on the Weinberg angle, $\Theta_{\mathrm{W}}$.

${ }^{2}$ The expression for the scattering cross section is given in eq (11) of ref. [2].
} 
where $|N\rangle$ represents a nucleon isospinor. Since the nucleon is an extended object form factors for this matrix elements are mandatory. The Lorentz covariant expressions for the (axial)vector current matrix elements of the flavor conserving components $(a=0,3,8)$ read

$$
\begin{aligned}
\left\langle N^{\prime}\left|V_{\mu}^{a}\right| N\right\rangle & =\bar{u}_{N}\left(p^{\prime}\right)\left[F_{1}^{a}\left(q^{2}\right) \gamma_{\mu}+F_{2}^{a}\left(q^{2}\right) i \sigma_{\mu \nu} \frac{q^{\nu}}{2 M}\right] u_{N}(p) \\
\left\langle N^{\prime}\left|A_{\mu}^{a}\right| N\right\rangle & =\bar{u}_{N}\left(p^{\prime}\right)\left[G_{A}^{a}\left(q^{2}\right) \gamma_{\mu} \gamma_{5}\right] u_{N}(p)
\end{aligned}
$$

where $u_{N}(p)$ denotes the proton Dirac spinor. The neutron form factors are obtained from isospin covariance. The linear combinations relevant for the processes under consideration are [3, 4]

$$
\begin{aligned}
F_{i}\left(q^{2}\right) & = \pm\left(\frac{1}{2}-\sin ^{2} \Theta_{\mathrm{W}}\right) F_{i}^{3}\left(q^{2}\right)-\sin ^{2} \Theta_{\mathrm{W}} F_{i}^{I=0}\left(q^{2}\right)-\frac{1}{2} F_{i}^{s}\left(q^{2}\right) \quad(i=1,2), \\
G\left(q^{2}\right) & =\mp \frac{1}{2} G_{A}^{3}\left(q^{2}\right)+\frac{1}{2} G_{A}^{s}\left(q^{2}\right)
\end{aligned}
$$

where $I=0$ and $s$ denote the non-strange and strange combinations of $a=0,8$, respectively. The upper (lower) sign refers to the proton (neutron). The vector form factors $F_{i}$ are more conveniently expressed in terms of electric and magnetic combinations

$$
G_{E}^{a}\left(q^{2}\right)=F_{1}^{a}\left(q^{2}\right)-\frac{q^{2}}{4 M^{2}} F_{2}^{a}\left(q^{2}\right) \quad \text { and } \quad G_{M}^{a}\left(q^{2}\right)=F_{1}^{a}\left(q^{2}\right)+F_{2}^{a}\left(q^{2}\right)
$$

The charge matrix is given by $\mathcal{Q}=\left(\lambda^{3}+\lambda^{8} / \sqrt{3}\right) / 2$ hence one may adopt the physically more transparent basis consisting of the electro-magnetic form factors of the nucleon, $G_{E, M}^{p, n}\left(q^{2}\right)$. Due to isospin covariance $G_{A}^{3}\left(q^{2}\right)$ is identical to the axial charge $G_{A}\left(q^{2}\right)$ of the nucleon extracted from neutron $\beta$-decay. The computation of these form factors in the Skyrme model with vector mesons will be reviewed in the next section.

\section{Nucleon form factors in the vector meson Skyrme model}

Our starting point is a three-flavor chirally invariant theory for pseudoscalar and vector mesons. The model Lagrangian also contains abnormal parity terms [7] to accommodate processes like $\omega \rightarrow 3 \pi$. A minimal set of symmetry breaking terms, which transform as $(\overline{3} \times 3+$ h.c. $)$ under the chiral group $S U_{L}(3) \times S U_{R}(3)$, is included [1] to account for different masses and decay constants. This effective theory contains topologically nontrivial static solutions, which are constructed by imposing ansätze in the isospin subgroup

$$
\xi_{\pi}(\boldsymbol{r})=\exp \left(\frac{i}{2} \hat{\boldsymbol{r}} \cdot \boldsymbol{\tau} F(r)\right), \quad \omega_{0}(\boldsymbol{r})=\omega(r) \quad \text { and } \quad \rho_{i, a}(\boldsymbol{r})=\frac{G(r)}{r} \epsilon_{i j a} \hat{r}_{j},
$$

\footnotetext{
${ }^{3}$ We omit the induced form factor of the axial current.
} 
while all other field components vanish. Here $\xi_{\pi}=\exp \left(i \boldsymbol{\pi} \cdot \boldsymbol{\tau} / 2 f_{\pi}\right)$ refers to the nonlinear realization of the pion fields. The radial functions are determined by extremizing the static energy functional subject to boundary conditions appropriate to the topological sector of winding number one. Motivated by the large $N_{C}$ studies of QCD these solitons are identified as the baryons with unit baryon number [17]. Unfortunately the field configuration (6) carries neither good spin nor flavor quantum numbers, hence an appropriate projection has to be performed. Also strange flavors are not yet excited. These two features are accounted for by introducing time dependent collective coordinates $A(t)$ for the (approximate) zero modes associated with the $S U(3)$ vector rotations: $\xi(\boldsymbol{r}, t)=A(t) \xi_{\pi}(\boldsymbol{r}) A^{\dagger}(t)$ and similarly for the vector meson nonet. Furthermore field components, which vanish classically like e.g. the kaons, are induced by the collective rotations. These induced fields turn out to be proportional to the angular velocities

$$
A^{\dagger} \dot{A}=\frac{i}{2} \sum_{a=1}^{8} \lambda^{a} \Omega^{a}
$$

and exist for both, rotations into strange $(a=4, . ., 7)$ and non-strange $(a=1,2,3)$ directions. In order to compute the induced fields ansätze are chosen, which are consistent with the parity as well as Lorentz and isospin structure of the considered mesons. Altogether eight real radial functions are introduced, which solve inhomogeneous linear differential equations derived from a variational principle to the moments of inertia [8]. The classical fields (6) act as source terms, which stem from the abnormal parity parts of the effective meson theory. The collective Lagrangian $L=L\left(A,\left\{\Omega^{a}\right\}\right)$ is extracted and the collective coordinates are quantized canonically [18]

$$
R_{a}=-\frac{\partial L\left(A,\left\{\Omega^{b}\right\}\right)}{\partial \Omega^{a}}
$$

providing a linear relation between the angular velocities $\Omega^{a}$ and the right generators of $S U(3), R_{a}$. The fact that these objects are operators is reflected by the commutation relations $\left[R_{a}, R_{b}\right]=-f_{a b c} R_{c}$, with $f_{a b c}$ being the structure constants of $S U(3)$. The resulting collective Hamiltonian may be diagonalized exactly 19 yielding the spectrum of the low-lying $\frac{1}{2}^{+}$and $\frac{3}{2}^{+}$baryons. In the absence of flavor symmetry breaking the eigenfunctions $\Psi(A)$ of this Hamiltonian are $S U(3)$ D-functions associated with a certain representation (e.g. the 8 for the nucleon). Once flavor symmetry breaking is included these D-functions become distorted reflecting the admixture of higher dimensional representations as for example the $\overline{\mathbf{1 0}}$ or $\mathbf{2 7}$.

Extending the action to account for electro-weak interactions allows one to derive covariant expressions for the (axial-) vector currents from the terms linear in the cor-

\footnotetext{
${ }^{4}$ The right generators of $S U(3)$ are identified in the body fixed system of the rotating soliton.
} 
Table I: Predicted form factors at zero momentum transfer. Three sets of parameters in the model Lagrangian are used. $(\times)$ : best fit to the baryon mass differences, $(*)$ : reproducing the experimental value for $G_{A}(0),(+)$ : best fit to $G_{A}(0), G_{M}^{p}(0)$ and $G_{M}^{n}(0)$. Furthermore the results associated with $S U(3)$ symmetric wave-functions are presented for the parameter set $(x)$.

\begin{tabular}{|c|ccccc|}
\hline & $G_{A}(0)$ & $G_{M}^{p}(0)$ & $G_{M}^{n}(0)$ & $G_{A}^{s}(0)$ & $G_{M}^{s}(0)$ \\
\hline$\times$ & 0.94 & 2.35 & -1.86 & -0.030 & -0.055 \\
$*$ & 1.25 & 3.23 & -2.86 & -0.015 & -0.035 \\
+ & 1.02 & 2.57 & -2.11 & -0.028 & -0.050 \\
\hline$S U(3)$ sym. & 0.88 & 2.48 & -1.54 & -0.058 & -0.559 \\
\hline Exp. & 1.25 & 2.79 & -1.91 & - & - \\
\hline
\end{tabular}

responding gauge fields. Substituting the above described ansätze and eliminating the angular velocities in favor of the generators (8) leaves the currents as linear combinations of radial functions and operators in the space of the collective coordinates $A$. The former are given in terms of the classical and induced profile functions of the meson fields. The matrix elements of the currents, which eventually provide the relevant form factors, are computed in two steps. Firstly, the radial functions are Fourier-transformed yielding the momentum dependence of the form factors in the Breit frame [20]. Secondly, an $S U(3)$ "Euler-angle" representation for $A(t)$ is employed [19] to parametrize the $S U(3)$ operators as well as the exact eigenfunctions $\Psi(A)$. This makes possible the evaluation of the spin and flavor parts in the matrix elements of the currents.

In ref. [8] the parameters of the model Lagrangian, which could not be determined from the meson sector, were adjusted to provide a best fit to the baryon mass differences. This has lead to reasonable, though not perfect, agreement for the electro-magnetic and axial form factors. The strange form factors, which are obtained by considering different flavor components of the currents, were predicted. In table [ 1 we display the results for zero momentum transfer together with results corresponding to parameter sets which improve on the axial charge of the nucleon and/or the magnetic magnetic moment of the nucleon. Also displayed are the predicted strange form factors at zero momentum transfer. Generally we find that all parametrizations predict the strangeness form factors to be negative and small in magnitude. In fig. 1 we display the predicted momentum dependence of the strange vector form factor $G_{S}\left(q^{2}\right)$. It is interesting to note that this quantity exhibits a local minimum at $q^{2} \approx 0.2 \mathrm{GeV}^{2}$ before dropping to zero at larger $q^{2}$. Apparently the frequently employed multipole parametrizations will not accurately reproduce this feature of $G_{S}\left(q^{2}\right)$. Kinematical corrections have not been incorporated here because they are not relevant for the momentum transfers of the present problem [21]. 
When $S U(3)$ symmetric wave-functions are employed to compute the spin and flavor parts of the matrix elements the strange magnetic moment increases by one order of magnitude as compared to the use of the exact eigenfunctions. This is not surprising since the use of these wave-functions assumes that strange quarks are as light as the non-strange ones. Obviously virtual strange degrees of freedom are easily excitable in that case.

The realistic amount of flavor symmetry breaking yields a small and negative value for the strange magnetic, even smaller than other estimates do [12, 22, 23, 24]. To some extend all these predictions seem to be contradictory to the preliminary results from the experiment at MIT-Bates: $G_{M}^{s}(0)=0.46 \pm 0.36 \pm 0.08 \pm 0.18$. The first erros is statistical, the second due to the background and the third takes account of uncertainties in the radiative corrections entering the analysis [25]. This measurement apparently favors a sizable positive value, however, due to large errors small negative values are not ruled out either. On the other hand, a large negative strange magnetic moment, as some calculations predict [26, 27], is unlikely. Estimates of the strange axial charge $G_{A}^{s}(0)$ are closely connected to the issue of the proton spin puzzle [5]. Employing flavor symmetry and using data from semi-leptonic hyperon decays results in $G_{A}^{s}(0) \approx-0.1$. When deviations from flavor symmetry are taken into account this number may easily be reduced and even a zero value is possible [9, 28.

\section{Results and discussion}

Before employing the above computed form factors to evaluate $R_{y}$ we will (in fig. 2) compare the old energy spectrum for the LAMPF neutrino beam with the new and more accurate one [6]. As can be observed when comparing the solid and dashed lines the energy distribution of the muon-neutrinos have not changed significantly. However, the new muon-antineutrino flux is different in shape and magnitude from the old one. Previously the latter was estimated by simply assuming it contributed $20 \%$ to the total $($ i.e. $\tilde{\nu}=\nu+\bar{\nu})$ neutrino beam. The improved spectrum for the antineutrinos is peaking at lower energies hence the yield induced by the antineutrinos is significantly decreased. The reason is that the extraction of $R_{y}$ requires a threshold of $E_{N}>60 \mathrm{MeV}$ for the energy of the knocked out nucleon to discriminate against events from elastic neutrino scattering on free protons.

We have next substituted the data of the improved spectra to predict $R_{y}$ as a function of the strange quark axial charge, $G_{A}^{s}(0)$ for different values of the strange quark magnetic moment $G_{M}^{s}(0)$. A dipole parametrization, which may not be very realistic $c f$. fig 11, has been employed for the momentum dependence [3]. In fig. 3 the results are displayed for both the quasi-elastic antineutrino- and neutrino-induced reactions on ${ }^{12} \mathrm{C}$. The range 
assumed for $G_{M}^{s}(0)$ is taken from the estimate of refs. [22, 23] [0. For the non-strange form factor also a dipole approximation has been adopted. As the reduction factor is the same for the proton and the neutron, the ratio $\sigma\left(\bar{\nu}, \bar{\nu}^{\prime} p\right) / \sigma\left(\bar{\nu}, \bar{\nu}^{\prime} n\right)$ is not effected when using the improved spectra. Hence the almost linear dependence on $G_{A}^{s}(0)$, which was previously found ( $c f$. fig. 4 in [4]), is recovered, although the absolute antineutrino induced cross sections have decreased by a factor of approximately 4.3. Since the interference terms contribute with opposite signs to the $\bar{\nu}$ - and $\nu$-induced reactions, $R_{y}$ exhibits a stronger increase with $G_{A}^{s}(0)$ for the $\bar{\nu}$ induced reaction than for the one associated with the neutrino.

Fig.3 also contains the prediction for $R_{y}$ obtained from the momentum dependent form factors predicted by the $S U(3)$ Skyrme model with vector mesons as outlined in the preceding section. While, for the case of antineutrino-induced reactions, the ratios are slightly different for the three parameters sets discussed above, they are practically indistinguishable for neutrino-induced scattering. As a consequence of the quite small value for $G_{M}^{s}(0)$ the prediction of this model is expected to be approximately given by the dashed lines in fig. 3. However, this is not quite the case since the model does not exactly reproduce the non-strange form factors, $c f$. table $\mathbb{\square}$.

In fig. \# we finally display $R_{y}(\tilde{\nu}=\nu+\bar{\nu})$ as obtained when both the neutrino and antineutrino yields are included. As a consequence of using the improved antineutrino spectrum, $R_{y}(\tilde{\nu})$ is not quite as sensitive to $G_{A}^{s}(0)$ as previously [3, 4] estimated. On the other hand the sensitivity on $G_{M}^{s}(0)$ is increased. Furthermore the negligibly small $\bar{\nu}$-flux causes the curves of fig. $2 b$ and fig. 4 to be identical within $1 \%$. From fig. 4 we deduce $1.1 \leq R_{y}(\tilde{\nu}) \leq 1.2$ as the prediction obtained from the Skyrme model with vector mesons. Adopting flavor symmetric wave-functions (i.e. $S U(3)$ D-functions) significantly increases this prediction, $R_{y}(\tilde{\nu}) \approx 1$.4. Thus an experimental determination of $R_{y}(\tilde{\nu})$ will in particular serve as a test of the quantization procedure proposed in ref. [19]. In this context it should be stressed that a precise measurement of $R_{y}$ will especially be suited to (dis)approve models for the baryons. The reason is that for this quantity the uncertainties inherited from the nuclear model will cancel almost completely. Barbaro et al. 13 have recently addressed this issue by comparing the single proton and neutron yields as well as their ratio $R_{y}$ in two extreme nuclear models with $G_{A}^{s}(0)$ being a parameter. It has then been observed that $R_{y}$ is almost independent of the nuclear model but very sensitive to $G_{A}^{s}(0)$, which, of course, is a prediction of the nucleon model. On the other hand the separate yields may significantly vary when $G_{A}^{s}(0)$ is kept fixed but different nuclear

\footnotetext{
${ }^{5}$ More recently a similar analysis which attempts to make contact with perturbative QCD has been performed in ref [24]. These authors consider their result $G_{M}^{s}(0)=-0.24 \pm 0.03$ as an upper bound (in magnitude). However, neither of these calculations incorporates the feature of flavor symmetry breaking.
} 
models are employed.

In our treatment strange degrees of freedom enter solely by virtual excitations of strange quark-antiquark pairs inside the nucleon. Let us also briefly coment on uncertainties which enter due to other effects. One may e.g. wonder whether or not the coupling of the leptonic current to the meson exchange currents between the constituents of the nucleus lead to additional contributions. This problem has been addressed by Musolf et al. 29. for the case of ${ }^{4} \mathrm{He}$. Although this nucleus is less complex than ${ }^{12} \mathrm{C}$ it represents a $J=0$ and $T=0$ nucleus as well. The exchange terms were observed to considerably improve on the agreement of the non-strange isoscalar form factor of ${ }^{4} \mathrm{He}$ with experiment (see fig. 2 of ref. [29]). Nevertheless they have turned out not to be relevant for momentum transfers less than approximately $500 \mathrm{MeV}$ for the strange vector form factor (see fig. 3 of ref. [29]). From the energy spectrum displayed in fig. 2 we hence conclude that our results will not significantly be modified by the contributions associated with the meson exchange currents. From a conceptual point the meson exchange terms are incorporated to satisfy current conservation for the many-body system as this requirement is not necessarily satisfied by the one-body current operator. One may estimate the corresponding uncertainties by employing various expressions for the current operator which are related by the continuity equation [30. For different excitations of ${ }^{12} \mathrm{C}$ Friar and Haxton 30 have compared the electric form factors predicted by various forms of the current operator with experimental data. They find that the form of the current operator which incorporates current conservation constraints only at zero momentum transfer $(q=0)$ but exhibits the correct high $q$ behavior contains at least some meson exchange effects. It is exactly this form which enters our calculation. The feature of correct high $q$ behavior is shared by neither the form of the current operator which is completely constrained by current conservation nor by the one which is not constrained at all.

We would also like to point out a possible source of uncertainty which is related to the measurement of the energy $E_{N}$ of the knocked out nucleon, which may be relevant for the interpretation of the experiments. Comparing the ratios $R_{y}$ in fig. 1 with the previous results (fig. 5 in ref. 四), we notice that the curves differ by a constant shift of approximately $15 \%$ in $R_{y}(\tilde{\nu})$. While for vanishing strangeness contributions to the form factors the present calculation (dashed line at $G_{A}^{s}(0)=0$ in fig. 4) gives the consistent value $R_{y}=1.0$, we extract $R_{y} \approx 0.85$ from fig. 5 in ref. 1 . This shift is caused by adopting different thresholds for the integrated neutron-yield. In ref. [4] $d \sigma / d E_{N}$ has been integrated over the energy of the emitted nucleon starting at $E_{N}=E_{p}=E_{n}+2.77 \mathrm{MeV}$ $>60 \mathrm{MeV}$ (i.e. as a consequence of the Coulomb-shift the energy threshold of the neutrons was taken to be $E_{n}^{0}=57.23 \mathrm{MeV}$ ), contrary to the results shown in fig. 2 and 
Table II: The ratios $R_{y}$ for neutrino-induced nucleon knockout on ${ }^{12} \mathrm{C}$ (and for $G_{A}^{s}(0)=$ $\left.G_{M}^{s}(0)=0\right)$ as a function of different thresholds for protons $\left(E_{p}^{0}\right)$ and neutrons $\left(E_{n}^{0}\right)$.

\begin{tabular}{|c|c|c|}
\hline$E_{p}^{0}[\mathrm{MeV}]$ & $E_{n}^{0}[\mathrm{MeV}]$ & $R_{y}(\nu)$ \\
\hline 60.0 & 60.0 & 1.004 \\
60.0 & 59.0 & 0.943 \\
60.0 & 61.0 & 1.069 \\
61.0 & 60.0 & 0.943 \\
61.0 & 59.0 & 0.885 \\
61.0 & 58.0 & 0.832 \\
61.0 & 61.0 & 1.004 \\
\hline
\end{tabular}

fig. 1 , where we have set the thresholds to $E_{N}^{0}=E_{p}^{0}=E_{n}^{0}=60 \mathrm{MeV}$. This crucial dependence of $R_{y}$ on the $E_{p^{-}}$and $E_{n}$-thresholds is linked to the steep decrease of the neutrino flux (fig. 2) between 70 and $200 \mathrm{MeV}$, which also causes the differential cross section $d \sigma / d E_{N}$ to strongly decrease with increasing $E_{N}$. We investigated this effect in more detail by slightly varying $E_{N}^{0}$ for protons and neutrons. The results for neutrinoinduced reactions are summarized in table प] (where we have set $G_{A}^{s}=G_{M}^{s}=0$, for simplicity). It is apparent that the experimentally determined value for $R_{y}$ crucially depends on an accurate measurement of the energy $E_{N}$ of the knocked out nucleon. A relative shift of only $1 \mathrm{MeV}$ between the thresholds $E_{p}^{0}$ and $E_{n}^{0}$ will cause an error for $R_{y}$ of $\approx 6 \%$. We find the same $\approx 6 \%$ error for non-zero $G_{A}^{s}$ and $G_{M}^{s}$ as well. It should, however, be remarked that the absolute value of the $E_{N}$-threshold has no influence, as long as $E_{p}^{0}$ and $E_{n}^{0}$ are identical. This independence was already pointed out previously, see fig. 3 of Ref. 四.

\section{Acknowledgements}

One of us (HW) acknowledges support by the Deutsche Forschungsgemeinschaft (DFG) under contracts We1254/2-1 and Re856/2-2. 


\section{References}

[1] M. Buballa, S. Drożdż, S. Krewald, and J. Speth, Ann. of Phys. 208, 346 (1991).

[2] E. Kolbe, K. Langanke, S. Krewald, and F. K. Thielemann, Nucl. Phys. A540, 599 (1992).

[3] G.T. Garvey, S. Krewald, E. Kolbe, and K. Langanke, Phys. Lett. B289, 249 (1992).

[4] G.T. Garvey, E. Kolbe, K. Langanke, and S. Krewald, Phys. Rev. C48, 1919 (1993).

[5] S. Brodsky, J. Ellis and M. Karliner, Phys. Lett. B206 (1988) 309;

J. Ellis and M. Karliner, Phys. Lett. B313 (1993) 131;

J. Schechter, A. Subbaraman and H. Weigel, Phys. Rev. D48 (1993) 339, and references therein.

[6] R.L. Burman, private communication.

[7] Ö. Kaymakcalan, S. Rajeev, and J. Schechter, Phys. Rev. D30 (1984) 594;

P. Jain, R. Johnson, Ulf-G. Meißner, N. W. Park and J. Schechter, Phys. Rev. D37 (1988) 3252.

[8] N. W. Park and H. Weigel, Phys. Lett. B268 (1991) 155; Nucl. Phys. A541 (1992) 453.

[9] R. Johnson, N. W. Park, J. Schechter, V. Soni, and H. Weigel, Phys. Rev. D42 (1990) 2998.

[10] N. W. Park, J. Schechter, and H. Weigel, Phys. Lett. B228, 420 (1989).

[11] P. Jain, R. Johnson, N. W. Park, J. Schechter, and H. Weigel, Phys. Rev. D 40 (1989) 855.

[12] For a review on estimates of the matrix element of the strange quark vector current see

M. J. Musolf, T. W. Donnelly, J. Dubach, S. J. Pollock, S. Kowalski, and E. J. Beise, Phys. Rep. 239 (1994) 1;

A comparison of results for this quantity in various models may also be found in

H. Forkel, M. Nielsen, X.-M. Jin, and T. Cohen, Phys. Rev. C50 (1994) 3108;

H. Weigel, Int. J. Mod. Phys. A11 (1996) 2419.

[13] M.B. Barbaro, A. De Pace, T.W. Donnelly, A. Molinari, and M.J. Musolf, "Probing nucleon strangeness with neutrinos: nuclear model dependencies", nucl-th/9605020.

[14] W.C. Louis (LSNC Collab.), spokesman, LAMPF proposal 1173.

[15] K. Nakayama, S. Drożdż, S. Krewald, and J. Speth, Nucl. Phys. A470, 573 (1987).

[16] R. Machleidt, K. Holinde, and Ch. Elster, Phys. Rep. 149, 1 (1987).

[17] E. Witten, Nucl. Phys. B160 (1979) 57.

[18] G. S. Adkins, C. R. Nappi, and E. Witten, Nucl. Phys. B228 (1983) 552.

[19] H. Yabu and K. Ando, Nucl. Phys. B301 (1988) 601;

N. W. Park, J. Schechter, and H. Weigel, Phys. Rev. D43 (1991) 869. 
[20] E. Braaten, S.-M. Tse and C. Willcox, Phys. Rev. Lett. 56 (1986) 2008; Phys. Rev. D34 (1986) 1482;

Ulf-G. Meißner, N. Kaiser, and W. Weise, Nucl. Phys. A466 (1987) 685.

[21] X. Ji, Phys. Lett. B254, 456 (1991).

[22] R.L. Jaffe, Phys. Lett. B229, 275 (1989).

[23] R.L. Jaffe and A. Manohar, Nucl. Phys. B337, 509 (1990).

[24] H.W. Hammer, Ulf-G. Meißner, and D. Drechsel, Phys. Lett. B367, 323 (1996).

[25] P. Souder, J. Schechter, private communication.

[26] D. Leinweber, "QCD Equalities for Baryon Current Matrix Elements", hep$\mathrm{ph} / 9512319$.

[27] H.-Y. Kim, T. Watabe, and K. Goeke, "Strange vector form factors of the nucleon in the SU(3) chiral quark-soliton model with the proper kaonic cloud", hep-ph/9606440.

[28] J. Lichtenstadt and H. J. Lipkin, Phys. Lett. B353, 119 (1995).

[29] M.J. Musolf, R. Schiavilla, and T.W. Donnelly, Phys. Rev. C50, 2173 (1994).

[30] J.L. Friar and W.C. Haxton, Phys. Rev. C31, 2027 (1985). 


\section{Figure captions}

Figure 1: The momentum dependent strange vector magnetic form factor $G_{M}^{s}$ in the $S U(3)$ vector meson Skyrme model (left panel). For completeness the strange axial vector form

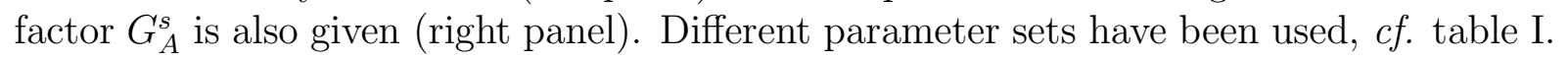

Figure 2: Comparison of the previous and updated (anti)neutrino spectra for the LAMPF decay-in-flight neutrino source. The solid, dotted and dashed lines refer to the updated neutrino, updated antineutrino and previous (anti) neutrino spectra, respectively. The antineutrino spectra are always scaled by a factor four.

Figure 3: Ratio of integrated proton-to-neutron yield for quasi-elastic antineutrino- (up-

per part) and neutrino-induced (lower part) reactions on ${ }^{12} \mathrm{C}$ as a function of $-G_{A}^{s}(0)$ for different values of $G_{M}^{s}(0)$ within the theoretically estimated regime [22, 23]. The symbols indicate the predictions of the $S U(3)$ Skyrme model with vector mesons, $c f$. table $\mathbb{\text { I. Their }}$ location on the horizontal axis reflects the associated prediction for $G_{A}^{s}(0)$.

Figure 4: Same as fig. 3, for the sum of antineutrino- and neutrino-induced yield. 

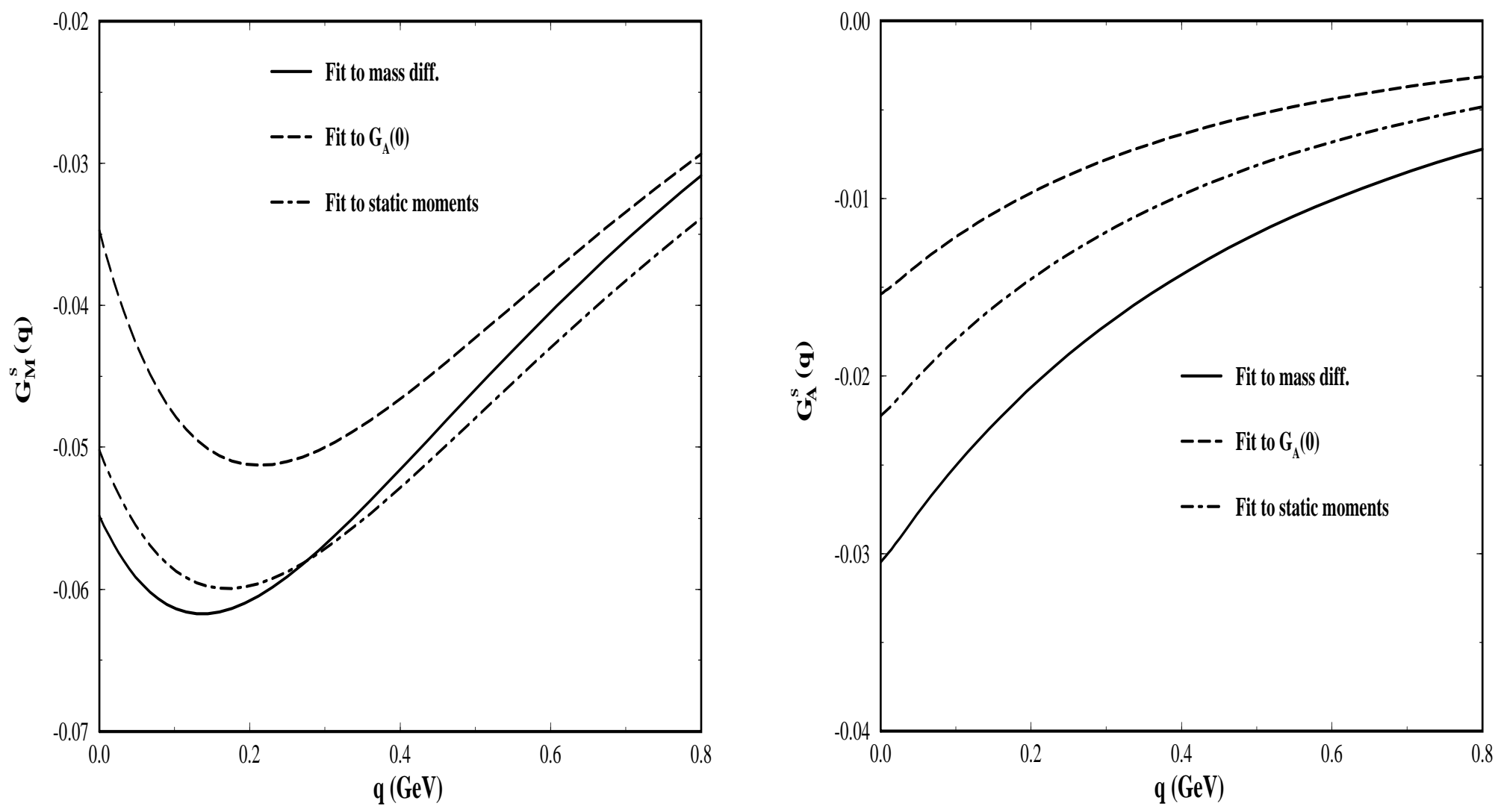


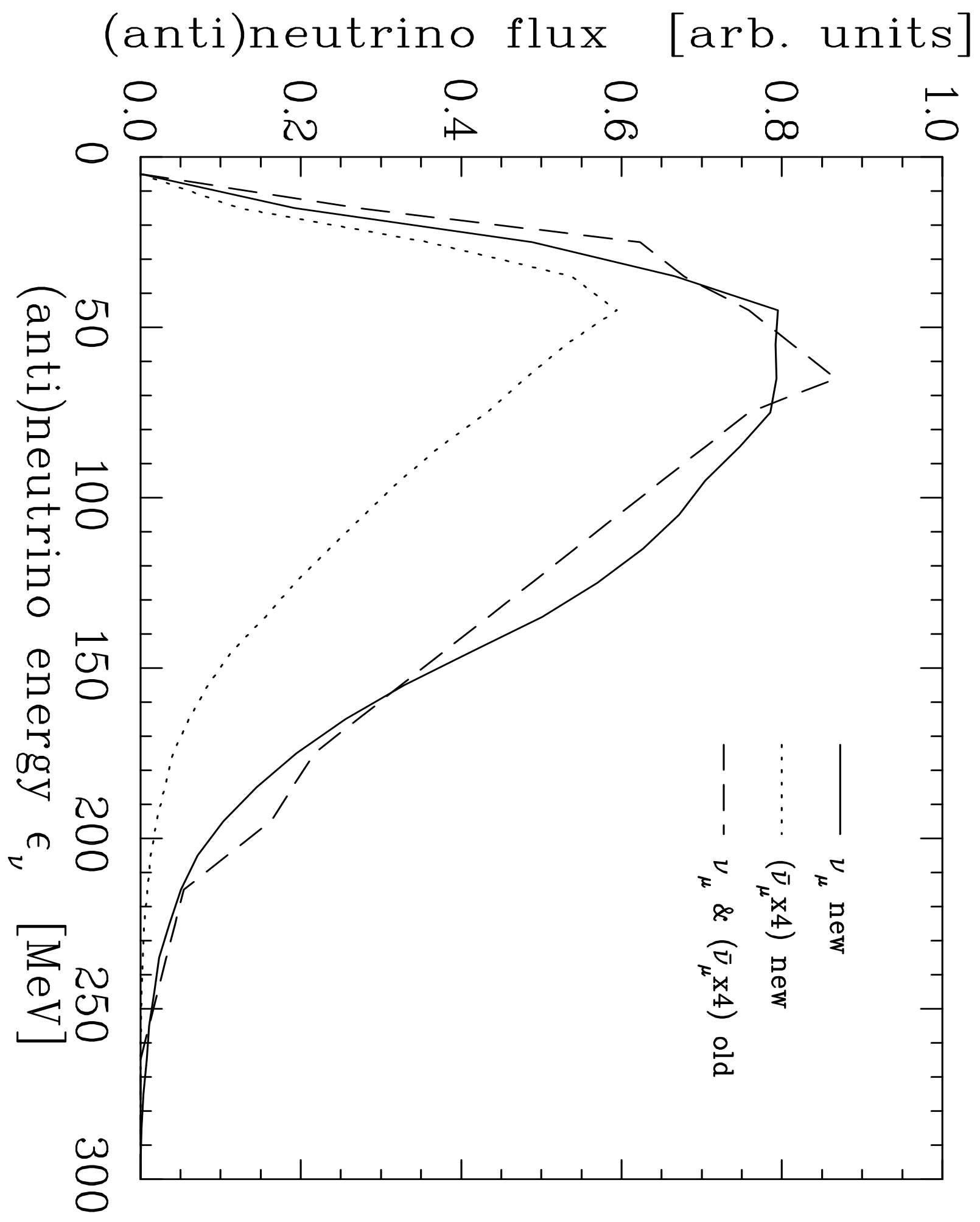



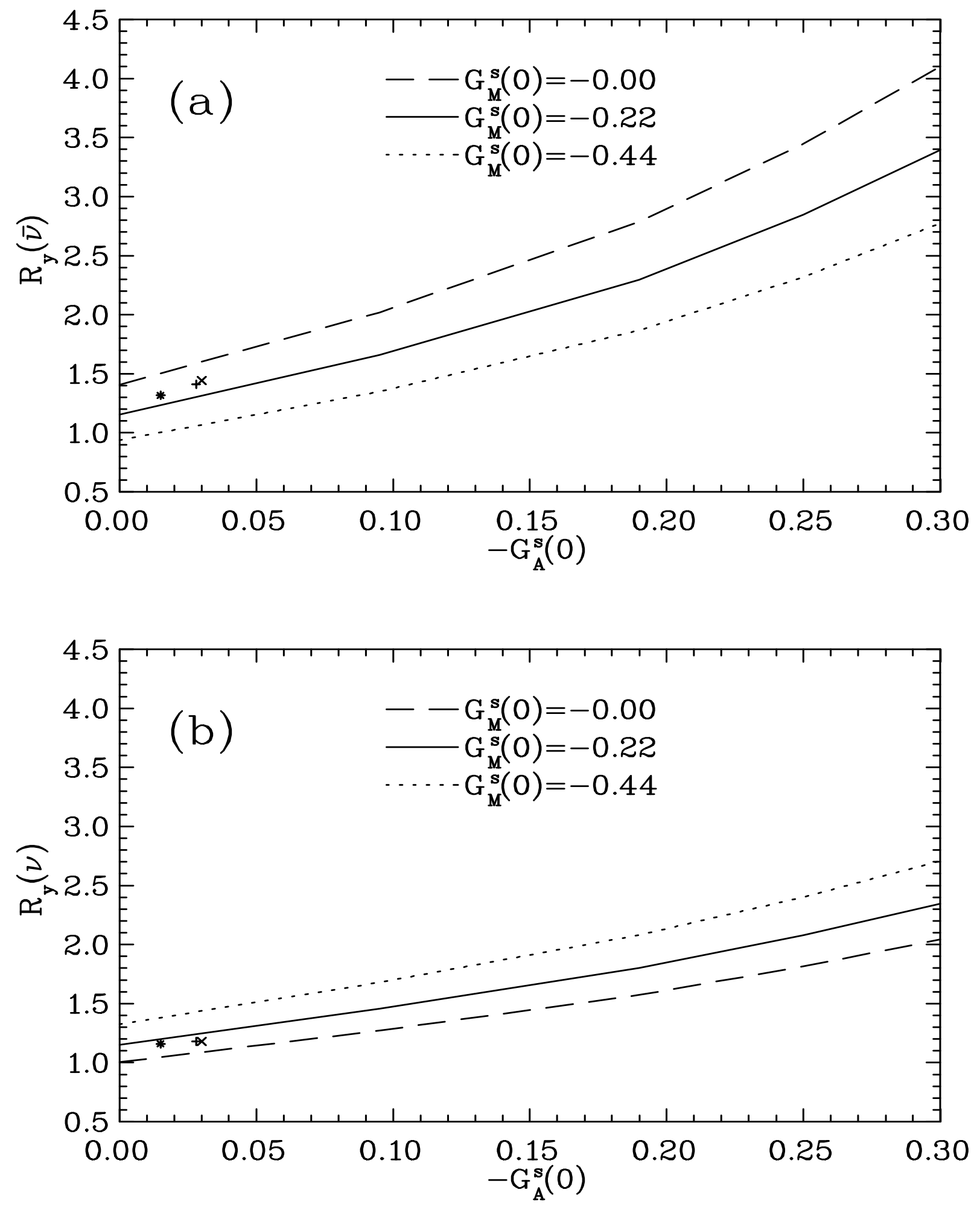


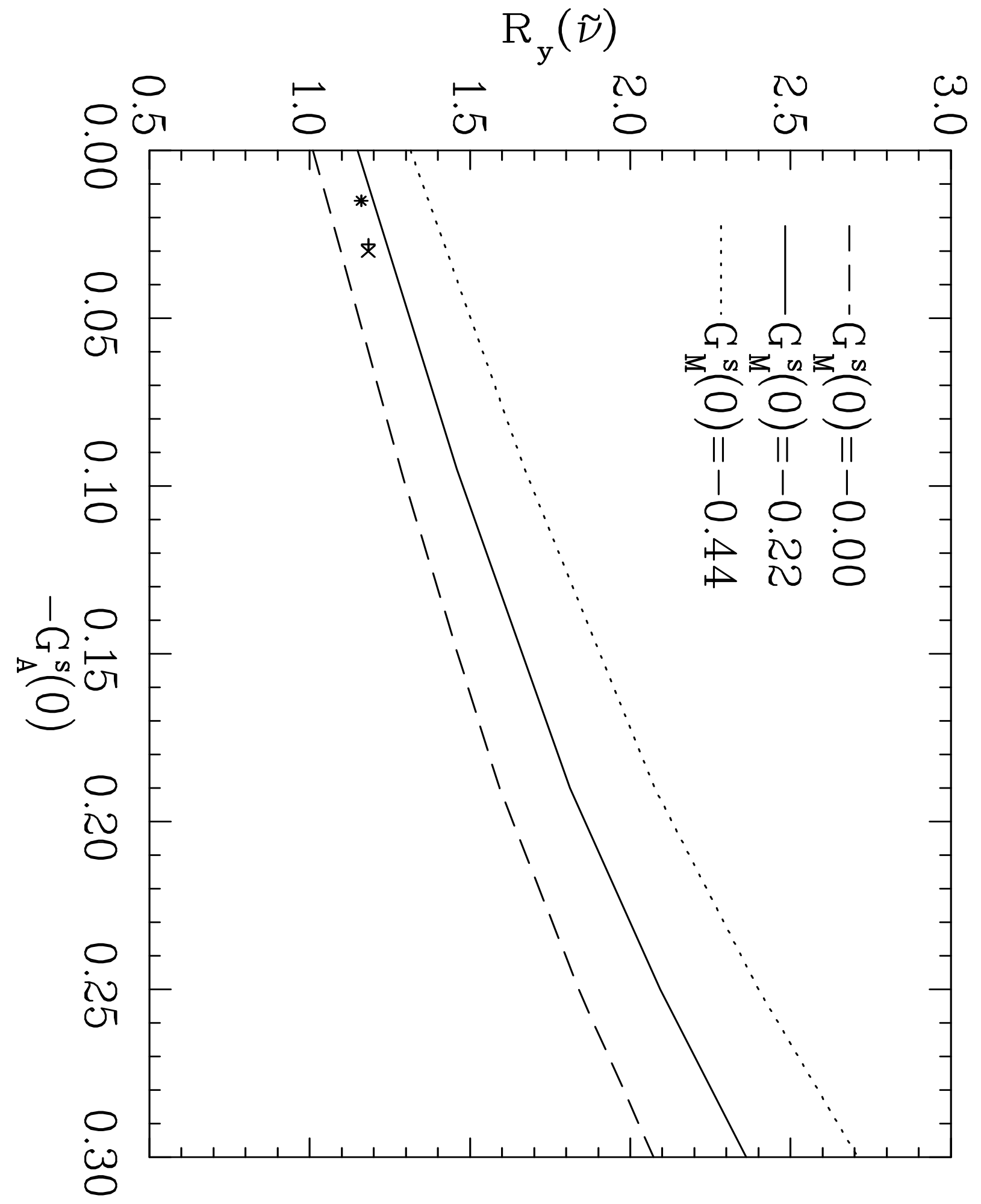

